\title{
The New Cryogenic Superfluid Gyroscope Based On Matter Wave Interference Effects
}

\author{
DENG Mao-lin ${ }^{1}$, ZHOU Yun-ting ${ }^{1, a}$, \\ ${ }^{1}$ Xichang Satellite Launch Center, XiChang, SiChuan 615000, China \\ azhouyt001@yahoo.com.cn
}

\begin{abstract}
Keywords: Cryogenic superfluid gyroscope; Extending measurement range; Amplitude locking; AC Josephson effect
\end{abstract}

\begin{abstract}
In this paper, the physical mechanism of the cryogenic superfluid gyroscope based on AC Josephson effect are analyzed, meanwhile, the implementation of the cryogenic superfluid gyroscope was brought up and the advantage of the sensitivity of the gyroscope is proved by theoretical derivation. A method to expand the measurement range based on amplitude locking technology are proposed, according to the problems of the insufficient range existing in the implementation of the cryogenic superfluid gyroscope based on AC Josephson effect when the information of corresponding angular velocity is extracted. Achievements of the study will be valuable reference to the simulation of cryogenic superfluid gyroscope.
\end{abstract}

\section{Introduction}

Depending on the physical effects, there are three different types of the cryogenic superfluid gyroscope. The cryogenic superfluid gyroscope based on quantum vortex effect, the cryogenic superfluid gyroscope based on DC Josephson effect and the cryogenic superfluid gyroscope based on AC Josephson effect [1-4]. In the study of the cryogenic superfluid gyroscope based on quantum vortex effect, quantized vortex is considered to be a micro-gyroscope formed by the rotation of the superfluid atoms. Due to the superfluid internal friction is almost negligible, the rotation of the quantized vortex can be considered without any resistance, thus the quantum vortex present the excellent rotational inertia [5]. But, the research of the cryogenic superfluid gyroscope based on quantum vortex effect is still in the stage of theoretical study and experimental verification, the theory has yet to be developed. Although the theory of the cryogenic superfluid gyroscope based on DC Josephson effect is more perfect, but its working temperature must be maintained at about $0.3 \mathrm{~K}$ [6]. The advantage of the sensitivity of the gyroscope is seriously damaged by the thermal noise when the cryogenic superfluid gyroscope is working in the ultra-low temperature environment. So, how to solve the problem of thermal noise has become a key to implement the cryogenic superfluid gyroscope based on DC Josephson effect. However, the effect of the thermal noise is successfully reduced in the physical mechanism of the AC Josephson effect as the working temperature is increased from $0.3 \mathrm{~K}$ to $2 \mathrm{~K}[7-8]$.

\section{Implementation}

In 2006, professor Packard's team of U.C. Berkeley discovered the induction relationship between the rotational angular velocity with the AC Josephson effect generated in the couple of weak links in the superfluid loop. This research achievements provide a scientific basis to the implementation of the cryogenic superfluid gyroscope based on AC Josephson effect [9]. The schematic diagram of the superfluid loop based on AC Josephson effect shown in Figure 1. 


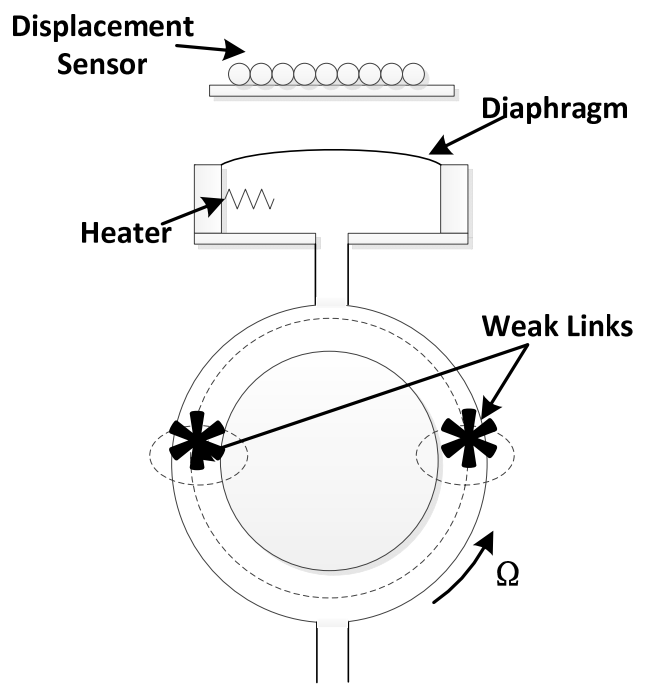

Figure .1 The schematic diagram of the superfluid loop based on AC Josephson effect

Figure .1 shows the couple of weak links (the nanoscale aperture arrays) are arranged symmetrically in the superfluid loop [10]. In a very short period of time, the temperature and pressure in the superfluid loop will be changed by the heater to creat a potential difference $\Delta \mu$

$$
\Delta \mu=m_{4}\left(\frac{\Delta P}{\Delta \rho}-s \Delta T\right)
$$

For superfluid $4 \mathrm{He}$, where $\mathrm{m}_{4}$ is the atomic mass of $4 \mathrm{He}, \rho$ is the total fluid density, $\mathrm{s}$ is an entropy per unit mass, and $\Delta \mathrm{p}$ and $\Delta$ Tare the pressure and temperature differences.

Because of the potential difference $\Delta \mu$, a phase difference is generated between each of the weak links. In this regime, one can define $\mathrm{I}_{1}=\mathrm{I}_{\mathrm{c}} \sin \Delta \phi_{1}$ describing the matter wave function on one side and $\mathrm{I}_{2}=\mathrm{I}_{\mathrm{c}} \sin \Delta \phi_{2}$ for the other. Here $\mathrm{I}_{\mathrm{c}}$ is the critical value of the mass current, $\Delta \phi_{1}=\Delta \phi_{2}=2 \pi \Delta \mu \mathrm{t} / \mathrm{h}$ is the phase difference, $\mathrm{h}$ is the Planck constant. The total mass current can be described

$$
I=I_{1}+I_{2}=2 I_{c} \sin \Delta \phi_{1}
$$

The matter wave propagate in the superfluid loop and a vibration is generated in the diaphragm

$$
\Delta x=\frac{1}{\rho A_{d}} \int_{0}^{T} I d t
$$

Here $\Delta x$ is the increment displacement of the diaphragm, $A_{d}$ is the area of the diaphragm. The external rotation presents a phase difference is generated by the Sagnac effect between each of the weak links and the mass current will be modulated by the angular velocity flux $\Omega$. A,where $\Omega$ is the external angular velocity, $\mathrm{A}$ is the area of the superfluid loop

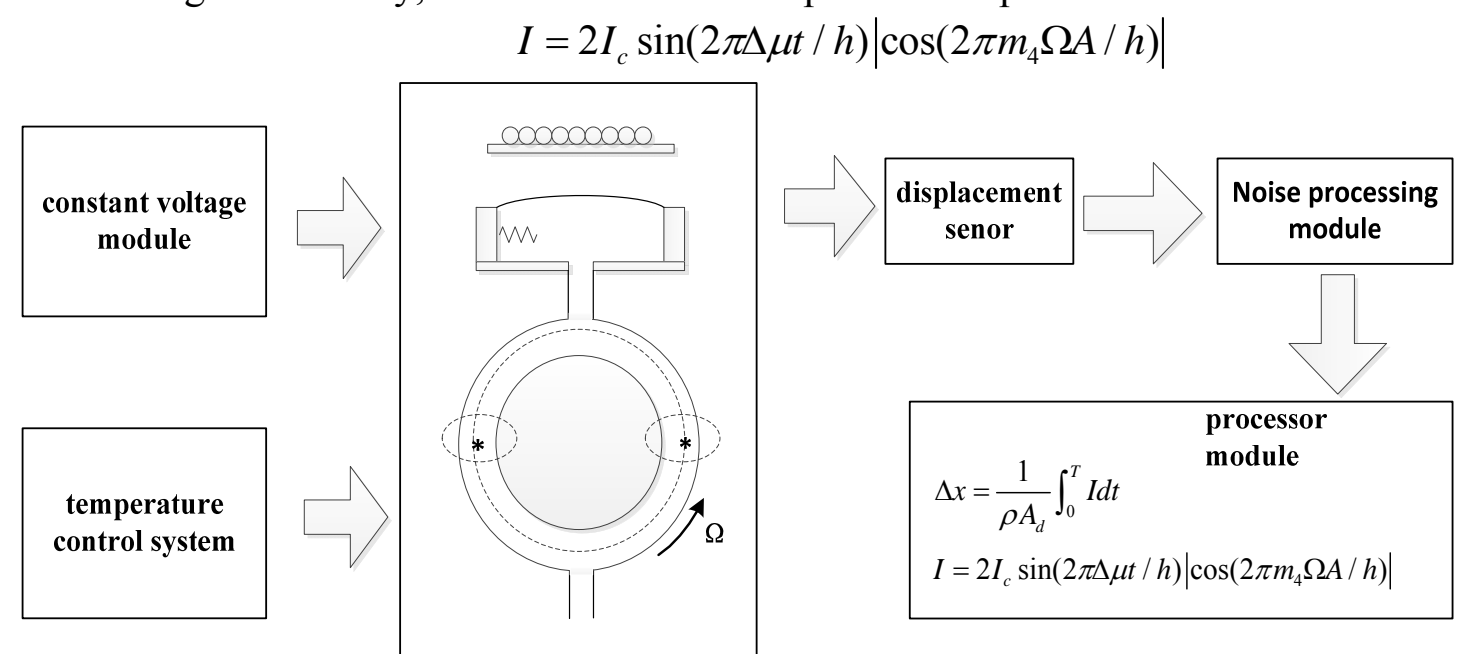

Figure .2 The implementation of the cryogenic superfluid gyroscope based on AC Josephson effect

Figure .2 shows the implementation of the cryogenic superfluid gyroscope, the temperature and 
pressure will be changed as a constant voltage is applied to the heater and the phase difference $\Delta \mu$ is generated between each of the weak links. The increment displacement of the diaphragm will be collected by the displacement senor. The denoising increment displacement is sent to the processor module and the external angular velocity will be calculated by the processor module. The working temperature will be maintained at $2 \mathrm{~K}$ by the temperature control system.

\section{Sensitivity}

The matter wave interference of the superfluid is similar to the light interference. If the rotation angular velocity is consistent with the vector direction of the sensitive axis, the angular velocity flux $\Omega$. A can be described to a scalar quantity form $\Omega$.A, the Sagnac phase shift of the light and the matter wave are given by Eq.(5)

$$
\Delta \phi_{\text {light }}=\frac{4 \pi}{\lambda c} \Omega A, \Delta \phi_{m v}=\frac{4 \pi}{\lambda_{m v} v} \Omega A
$$

At the same external angular velocity $\Omega$, the relationship between the matter wave phase shift and the light phase shift can be defined.

$$
\Delta \phi_{m v}=\frac{4 \pi}{\lambda_{m v} v}\left(\frac{4 \pi \Omega A}{\lambda c}\right)=\frac{\lambda c}{\lambda_{m v} v} \Delta \phi_{\text {light }}
$$

Because the wavelength of the matter wave only relate with the particle momentum $\left(\lambda_{\mathrm{mv}}=\right.$ $\mathrm{h} / \mathrm{mv}$ ), so Eq.(6) can be redefined.

$$
\Delta \phi_{m v}=\frac{\lambda m c}{h} \Delta \phi_{\text {light }}
$$

Here $h$ is the Planck constant $\left(6.69 \times 10^{-27} \mathrm{~J} . \mathrm{s}\right), \mathrm{m}$ is the atomic mass of $4 \mathrm{He}(6.626 \times$ $\left.10^{-34} \mathrm{~kg}\right), \mathrm{c}$ is the lightspeed $\left(3 \times 10^{8} \mathrm{~m} / \mathrm{s}\right)$. At the same external angular velocity $\Omega$, the phase difference of the matter wave is $10^{9}$ times than the phase difference of the light.

\section{Extending measurement range}

Eq.(6) shows there is a corresponding periodic relationship between the vibration displacement of the diaphragm and the external angular velocity when the external angular velocity is a periodic signal. The amplitude of the vibration signal caused by he AC Josephson effect in the weak link will be modulated by the phase changes of the external phase rotation. Because the value of the parameter $\left(\mathrm{m}_{4} / \mathrm{h}\right)$ is large, so the cryogenic superfluid gyroscope can detect very small angular velocity flux $\Omega$.A, and then the external angular velocity $\Omega$ can be calculated. However, due to the presence of a periodic signa, there is a single value range restriction $(n \pi, n \pi+\pi / 2)$ when the external angular velocity $\Omega$ is calculated. If the rotation angular velocity is larger than $\pi / 2$ or much larger than $\pi / 2$, the angular velocity information of the periodic signa can be described.

$$
2 \pi m_{4} \Omega \frac{A}{h}=n \frac{\pi}{2}+\Delta \phi_{\text {margin }}
$$

where $\mathrm{n}$ is an integer, $\Delta \phi_{\text {margin }}$ is a phase margin( the value greater than 0 and less than $\pi / 2$ ). Eq.(6) shows the external angular velocity $\Omega$ calculated by the vibration displacement of the diaphragm is $\Delta \phi_{\text {margin }}$, due to the impact of the periodic signal, and the difference value is $n \pi / 2$ between the result and the actual value,so how to solve the single value range restriction and extend the measurement range of the cryogenic superfluid gyroscope is a key issue to achieve the cryogenic superfluid gyroscope based on AC Josephson effect. 


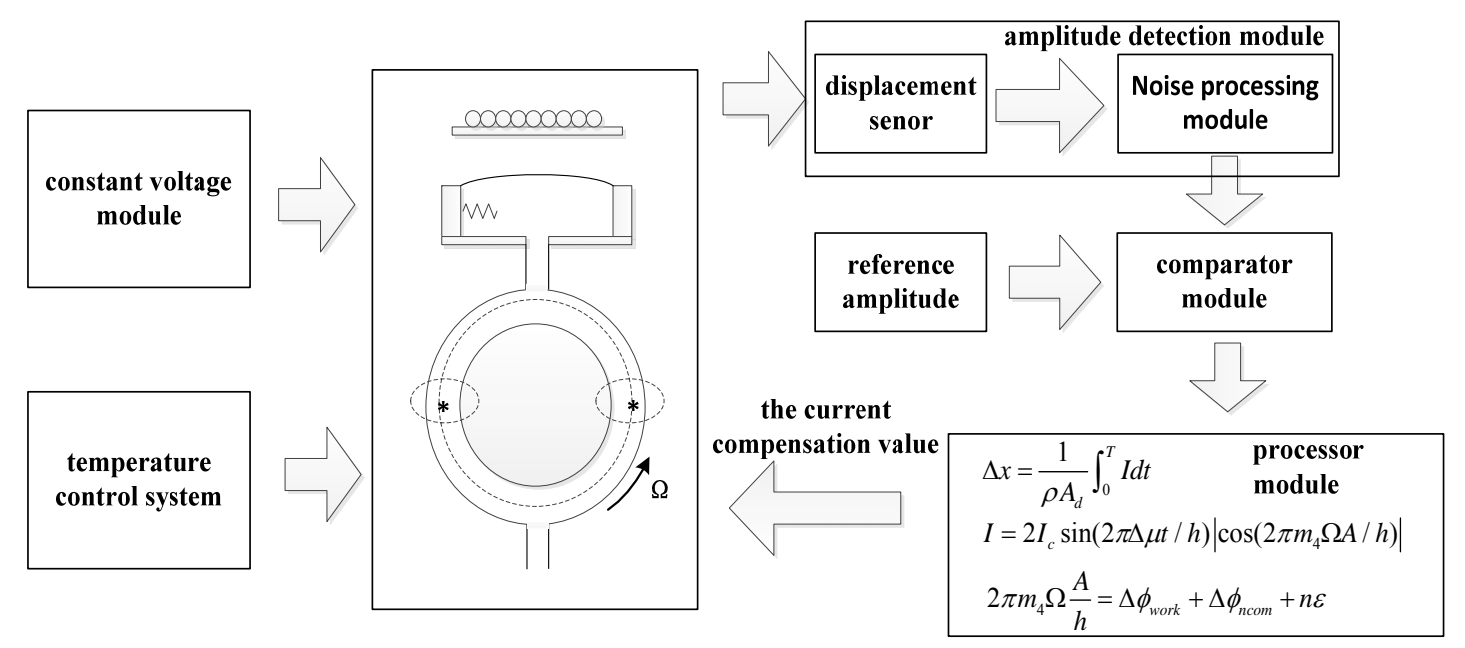

Figure 3 . The schematic diagram of the amplitude locking method

Scholars have proposed the amplitude locking method which is similar to the phase locking method of the superconducting quantum interference device to extend the measurement range of the cryogenic superfluid gyroscope. The main idea is to use a thermal feedback signal to compensate the phase difference caused by the external angular velocity $\Omega$, and the amplitude of the diaphragm will be controlled in a fixed working point or in a monotonic interval with the excellent linearity.

Figure .3 shows the schematic diagram of the amplitude locking method.First, set up a working point for the cryogenic superfluid gyroscope before the system is running, the phase of the working point is determined by the external angular velocity $\Omega$ and the compensation values and the reference amplitude of the system is the amplitude of the working point. In the first step ,the most important thing is to set up the working piont in the middle of the best linearity range. Second, make the system continue to work at the working poin, and collect the current amplitude of the diaphragm by the amplitude detection module[11]. The amplitude difference between the current amplitude and the reference amplitude will be calculated by the comparator module, subsequently, the corresponding phase difference is calculated by the processor module and the current compensation values will be defined as the sum of the current phase difference and the previous value. Finally, the current compensation value should be fed back to the superfluid loop by the heater for compensating the phase difference caused by the external angular velocity $\Omega$. If the rotation angular velocity is consistent with the vector direction of the sensitive axis, Eq.(8) can be redefined.

$$
2 \pi m_{4} \Omega \frac{A}{h}=\Delta \phi_{\text {work }}+\Delta \phi_{\text {com }}
$$

Here, $\phi_{\text {work }}$ is the phase of the working point set before the system is running, $\Delta \phi_{\text {com }}$ is the current compensation phase.

In addition, according to research result of the Berkeley Physics Laboratory, the phase difference compensated by the heater must be less than $500 \pi[12]$. Because the temperature of the superfluid loop will be raised too high to occur quantum disorder and damage the AC Josephson effect in the superfluid loop when the phase difference compensated by the heater is more than $500 \pi$. If the rotation angular velocity is consistent with the vector direction of the sensitive axis and the $\mathrm{A}$ is $0.001 \mathrm{~m}^{2}$, the upper limit of the compensation value is defined in Eq.(10)

$$
2 \pi m_{4} \Omega \frac{A}{h} \leq 500 \pi
$$

Eq.(10) shows the maximum of the external angular velocity $\Omega$ detected by the cryogenic superfluid gyroscope $\Omega \leq 0.025 \mathrm{rad} / \mathrm{s} \approx 1.4\left(^{\circ}\right) / \mathrm{s}$, the measurement range of the external angular velocity $\Omega$ extended by the amplitude locking method is still small and doesn't have use value. We add a compensation threshold value in the system (the compensation threshold value is slightly less than $500 \pi$ ) to solve this problem. If the current compensation value $\Delta \phi_{\text {com }}$ is more than the compensation threshold value, the current compensation value will be redefined as $\Delta \phi_{\text {ncom }}$ which is the difference between the current compensation value $\Delta \phi_{\text {com }}$ and the compensation threshold 
value , and the number of times the current compensation value minus the compensation threshold value need to be recorded.Here, nis the number of times the current compensation value minus the compensation threshold value, $\varepsilon$ is the compensation threshold value.

$$
2 \pi m_{4} \Omega \frac{A}{h}=\Delta \phi_{\text {work }}+\Delta \phi_{\text {ncom }}+n \varepsilon
$$

\section{Summary}

The cryogenic superfluid gyroscope based on AC Josephson effect is a new generation of high-precision gyroscopes and how to solve the single value range restriction and extend the measurement range of the cryogenic superfluid gyroscope is a key issue to achieve the cryogenic superfluid gyroscope based on AC Josephson effect. In this paper, related issues of the cryogenic superfluid gyroscope on AC Josephson effect have been studied and discussed in depth. The following aspects have been come to conclusions specifically.

1. The physical mechanism of the cryogenic superfluid gyroscope based on AC Josephson effect are analyzed.

2. The advantage of the sensitivity of the gyroscope is proved by theoretical derivation.

3. A method to expand the measurement range based on amplitude locking technology are proposed to extend the measurement range of the cryogenic superfluid gyroscope.

\section{References}

[1] J. C. Davis and R. E. Packard, The Siperfluid Helium Gyroscope: An Emerging Technology for Earth Rotation Studies, Proc. of the AGU Chapman Conference on Geodetic VLBI: Monitoring Global Change, 1991, 137:49.

[2] E. Hodby, S. A. Hopkins, G. Hechenblaikner et al. Expermental Obserbation of a Superfluid Gyroscope in a Dilute Bose-Einstein Condensate. Physics Review Letters, 2003, 91(9):090303.1090303.4.

[3] David W Hallwood, Keith Burnett, Hacob Dunningham, Macroscopic Superpositions of Superfluid Flows, New Journal of Physics, 2006, 8(180):1-8.

[4] O. Avenel, Yu. Mukharsky, and E. Varoquaux, Superfluid Gyrometers, Journal of Low Temperature Physics, 2004, 135(5):745-722.

[5] S. Stringari. Superfluid Gyroscope with Cold Atomic Gases. Physics Review Letters, 2001,86(21):4725-4728.

[6] N. Bruckner, Richard Packard, Detection of absolute rotation using superfluid 4He. Low Temp. Phys.,1998, 24(2):102-104.

[7] Y. Sato, A. Joshi, and R. Packard, Direct Measurment of Quantum Phase Gradients in Superfluid 4He Flow, Physical Review Letters, 2007, 98:195302.1-195302.3.

[8] Y. Sato, R E Packard, Superfluid helium quantum interference device: physics and applications. REPORTS ON PROGRESS IN PHYSICS, 75:016401.1-016401.27.

[9] Hoskinson Emile, Sato Yuki, Packard Richard. Superfluid 4He interferometer operating near 2K. Physical Review B, 2006, 74(10):100509.1-100509.8.

[10]R. Packard, Superfluid Helium 4 Weak Links and Application. (2008-08-15), http//www.phsics.berkeley.edu/research/packard/current_research/4He/4He_main.html.

[11]SRINATH S. A review of the SQUID handbook: fundamentals and technology of SQUIDs and SQUID systems[J]. Meterials \& Manufacturing Processes, 2006, 21(5):583-583.

[12]Y. Sato, A. Joshi, and R. Packard, Flux Locking A Superfluid Interferometer, Physical Review Letters, 2007, 91(7):074107.1-074107.3. 